\title{
ARTICLE Striatal activation to monetary reward is associated with alcohol reward sensitivity
}

\author{
Milena Radoman (iD), Natania A. Crane ${ }^{1}$, Stephanie M. Gorka ${ }^{2}$, Jessica Weafer ${ }^{3}$, Scott A. Langenecker ${ }^{4}$, Harriet de Wit ${ }^{5}$ and K. Luan Phan ${ }^{2}$
}

One well-known phenotypic risk factor for the development of alcohol use disorder is sensitivity to the rewarding effects of alcohol. In the present study, we examined whether individuals who are sensitive to alcohol reward are also sensitive to nondrug rewards, thereby reflecting a broader individual difference risk factor. Specifically, we tested the hypothesis that subjective response to acute rewarding effects of alcohol would be related to neural activation during monetary reward receipt relative to loss (in the absence of alcohol). Community-recruited healthy young social drinkers $(N=58)$ completed four laboratory sessions in which they received alcohol $(0.8 \mathrm{~g} / \mathrm{kg})$ and placebo in alternating order under double-blind conditions, providing self-report measures of subjective response to alcohol at regular intervals. At a separate visit 1-3 weeks later, they completed a reward-guessing game, the 'Doors' task, during fMRI in a drug-free state. Participants who reported greater motivation (i.e., wanting) to consume more alcohol after a single moderate dose of alcohol also exhibited greater neural activation in the bilateral ventral caudate and the nucleus accumbens during reward receipt relative to loss. Striatal activation was not related to other subjective ratings including alcohol-induced sedation, stimulation, or pleasure (i.e., feeling, liking). Our study is the first to show that measures of alcohol reward are related to neural indices of monetary reward in humans. These results support growing evidence that individual differences in responses to drug and nondrug reward are linked and together form a risk profile for drug use or abuse, particularly in young adults.

Neuropsychopharmacology (2021) 46:343-350; https://doi.org/10.1038/s41386-020-0728-6

\section{INTRODUCTION}

Heavy alcohol consumption elevates the risk of numerous public health and socioeconomic problems and is the third leading lifestyle-related cause of death [1-3]. Understanding the etiology of problematic alcohol use is therefore crucial to improving early detection, prevention, and treatment strategies. One individual vulnerability risk factor that may increase the propensity for hazardous drinking is subjective response to the pharmacological effects of alcohol [4, 5]. To date, numerous studies have shown that individuals with greater stimulating, rewarding (liking, wanting) responses as well as lower sedative responses to alcohol were more likely to experience drinking problems in the future and develop alcohol use disorder (AUD; [6-8]). In light of this, a growing focus of research has been identifying neurobiological risk factors that contribute to differences in sensitivity to the rewarding effects of alcohol for the purpose of developing brainbased therapeutic interventions.

One way alcohol produces its rewarding effects is through the activation of the mesolimbic dopaminergic reward circuitry [9-12]. The drug stimulates midbrain dopamine projections to the ventral striatum, including the nucleus accumbens (NAcc), which is especially important in regulating the subjective rewarding effects of alcohol [13-15]. Single doses of alcohol increase activation of the NAcc, possibly by increasing the firing rate of dopaminergic neurons resulting in greater extracellular dopamine release [16-21]. There is also evidence that these alcohol-induced changes in the striatum are related to self-report measures of subjective intoxication. For example, using positron emission tomography, researchers have shown that individuals with greater self-reported feelings of 'intoxication' and 'high' also displayed greater alcohol-related increase in striatal dopamine release $[22,23]$. In addition, functional magnetic resonance imaging (fMRI) studies involving acute intravenous infusion of alcohol have found that greater alcohol-induced activation in the ventral striatum and the NAcc is correlated with subjective feelings of euphoria and stimulation [24-26]. Collectively, these studies suggest that the rewarding effects of acute alcohol intoxication may be related to activation of the ventral striatum, perhaps mediated via extracellular dopamine release.

Dopamine signaling, and striatal activation in particular, are also involved in the processing of nondrug rewards [27, 28]. For instance, both primary rewards such as liquids, food, and sexual stimuli, as well as secondary rewards, such as money, activate the ventral striatum, and the NAcc [29-42]. The observed similarities in neural response to drug and nondrug rewards align with the 'incentive-salience' theory, which posits that striatal dopamine release enhances saliency of reward-related stimuli, thereby increasing individual's motivation to seek and acquire rewards,

\footnotetext{
${ }^{1}$ Department of Psychiatry, University of Illinois at Chicago, 1601 W Taylor Street, Chicago, IL 60612, USA; ${ }^{2}$ Department of Psychiatry and Behavioral Health, Ohio State University, 1670 Upham Drive, Columbus, OH 43205, USA; ${ }^{3}$ Department of Psychology, University of Kentucky, 171 Funkhouser Drive, Lexington, KY 40506, USA; ${ }^{4}$ Department of Psychiatry,

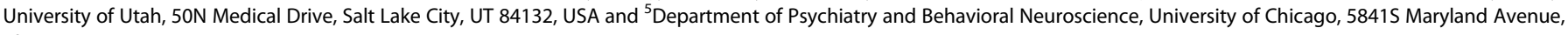
Chicago, IL 60637, USA

Correspondence: Milena Radoman (mradom3@uic.edu)

These authors contributed equally: Harriet de Wit, K. Luan Phan
}

Received: 17 February 2020 Revised: 8 May 2020 Accepted: 28 May 2020

Published online: 6 June 2020 
both drugs of abuse or nondrug incentives $[43,44]$. Related to this, emerging evidence suggests that sensitivity of reward processes in response to alcohol and other drug-related cues may be intricately associated with sensitivity to nondrug rewards [45-49]. That is, in a natural environment that often offers many concurrent rewarding opportunities, individuals with a greater sensitivity to reward more broadly may also be more likely to engage in activities that involve salient nondrug incentives. For example, they may be more likely to attend a party where there are numerous social and other rewards (e.g., poker for monetary reward), and where alcohol (drug reward) is freely available [50]. It is therefore hypothesized that reward sensitivity may extend beyond just drugs of abuse to other forms of reward, thereby reflecting a broader individual difference risk factor, perhaps due to individual variability in brain reward function.

Consistent with this hypothesis, we recently showed that greater activation in mesolimbic reward regions to monetary reward anticipation in a drug-free state was associated with greater subjective rewarding effects of acute d-amphetamine $[51,52]$. Using a design similar to the one described here, healthy young adults participated in behavioral sessions in which they received amphetamine and placebo, followed by a drug-free imaging session to assess neural response to monetary reward.

In the present study we extended these results to alcohol by examining the relationship between neural activity during nondrug (monetary) reward (in the absence of alcohol) and sensitivity to the subjective rewarding effects of alcohol, among healthy, nondependent social drinkers. Based on evidence that reward anticipation and receipt are two distinct reward processes [53], here we analyzed only responses to reward receipt, and plan to examine other aspects of the reward response separately. Neural response was assessed using fMRI while participants performed a monetary reward-guessing game, the 'Doors' task, in a drug-free state. We focused our analyses on the mesolimbic reward circuitry, particularly the striatum. Subjective rewarding effects of alcohol were assessed using standardized self-report questionnaires, following acute oral doses of alcohol and placebo administered under double-blind conditions. We hypothesized that greater neural activation during monetary reward receipt relative to loss would be associated with greater rewarding response to alcohol relative to placebo.

\section{MATERIALS AND METHODS}

\section{Participants}

Participants were right-handed, healthy social drinkers (aged 21-29) recruited from the local Chicago community and nearby college campuses through online and printed advertisements. Eligible participants consumed on average 7-30 standard drinks per week (e.g., $12 \mathrm{oz}$ beer or $1.5 \mathrm{oz}$ liquor), and had at least one heavy drinking episode (i.e., four or more drinks in one sitting for women, five or more for men) in the past month $[54,55]$. These drinking criteria ensured that participants could tolerate the alcohol dose administered in the study and that the study included some heavier drinkers who might be at risk for developing AUD. Additional inclusion criteria included body mass index between 19 and 26, at least a high school education, and English fluency. Participants were excluded if they had a current or past year DSM-5 diagnosis [56], lifetime history of substance use disorder or ADHD, history of psychosis, a serious medical condition, abnormal electrocardiogram, smoked $>5$ cigarettes per day, used medications daily (other than birth control), or if they were pregnant, lactating, or planning to become pregnant in the next 3 months. Additional exclusion criteria included night shift work, positive urine drug screen on any study session, and contraindications for $\mathrm{fMRl}$, including metal in the body or history of claustrophobia. Of the 75 individuals initially enrolled, 4 participants dropped out of the study before completing all
Table 1. Demographics and clinical characteristics $(N=58)$.

\begin{tabular}{ll}
\hline & Mean (SD) or \% \\
\hline Demographics & \\
Age (years) & $24.4(2.6)$ \\
Sex (\% female) & $48.3 \%$ \\
Ethnicity (\% Hispanic) & $10.3 \%$ \\
Race & \\
$\quad$ White & $55.2 \%$ \\
Black & $24.1 \%$ \\
Asian & $6.9 \%$ \\
American Indian or Alaskan Native & $1.7 \%$ \\
$\quad$ Other or unknown & $12.1 \%$ \\
Current substance use & \\
Alcohol (drinks/week) & $12.6(5.8)$ \\
Cigarettes (per day) & $0.35(1.0)$ \\
Caffeine (cups/day) & $2.0(1.8)$ \\
Marijuana (times/month) & $10.9(15.1)$ \\
Lifetime substance use (\% ever used) & \\
Marijuana & $65.5 \%$ \\
Hallucinogens & $48.2 \%$ \\
Stimulants & $51.7 \%$ \\
Opiates & $36.2 \%$ \\
MDMA & $53.4 \%$ \\
Sedatives & $32.8 \%$ \\
Subjective response to drug & \\
BAES & \\
Stimulation PCDS & \\
Sedation PCDS & $7.9(13.8) ;$ range: -23.5 to +42.0 \\
DEQ & \\
Feel drug PCDS & $48.5(21.1) ;$ range: +1.0 to +97.5 \\
Like drug PCDS & $47.7(22.8) ;$ range: +9.0 to +95.5 \\
Want more drug PCDS & $41.0(29.9)$; range: -17.5 to +100 \\
\hline BAES Biphange: -32.0 to +47.0 \\
\hline
\end{tabular}

BAES Biphasic Alcohol Effects Scale, DEQ Drug Effects Questionnaire, PCDS peak change difference score [alcohol-placebo].

laboratory visits, 1 was missing imaging data, and 12 participants were excluded from analyses for significant movement (as described below). The final sample included 58 individuals (see Table 1 for detailed demographics and clinical characteristics).

\section{Study design and procedures}

Participants completed an initial screening and orientation visit during which they provided written informed consent and were familiarized with laboratory procedures and study protocol. They then completed four beverage-sampling sessions at University of Chicago (UC), followed by a separate fMRI visit at University of Illinois at Chicago (UIC) 1-3 weeks later. During the fMRI session they completed a reward-guessing game, the 'Doors' task, in a drug-free state. Participants were asked to abstain from drugs, including alcohol, for $24 \mathrm{~h}$ prior to each visit, which was verified by self-report, breath alcohol (Alco-Sensor III; Intoximeters, St. Louis, $\mathrm{MO}$ ), and urine screens (Instant Drug Test Cup; CLIAwaived, San Diego, CA). Urine was tested for tetrahydrocannabinol, cocaine, amphetamine, morphine, phencyclidine, barbiturates, benzodiazepines, MDMA, oxycodone, and buprenorphine. The Institutional Review Boards at UC and UIC approved the study procedures. After completing all sessions, participants were debriefed and monetarily compensated for their time.

Alcohol challenge sessions

Detailed methodology has been published elsewhere [57] and is summarized here. The alcohol/placebo challenge sessions were 
conducted in a comfortable living room like setting, from 3:00 to 8:00 p.m., separated by 2-7 days. During these four sessions participants received alcohol and placebo in alternating order, under double-blind conditions, with the initial drug randomized. Alcohol and placebo were each administered twice to minimize the influence of day-to-day variability, but for the present analysis the data from the two sessions were combined [58, 59]. For blinding purposes, participants were told they would receive one of the following: stimulant, sedative, alcohol or placebo. Upon arrival to the laboratory subjects provided urine and breath samples for verification, and then completed prebeverage mood questionnaires. At 3:30 p.m., participants consumed beverages containing $0.8 \mathrm{~g} / \mathrm{kg}$ ethanol (divided into four servings of $0.2 \mathrm{~g} / \mathrm{kg}$ each) or a matching placebo. The ethanol dose was $0.8 \mathrm{~g} / \mathrm{kg}$ for men, and $0.7 \mathrm{~g} / \mathrm{kg}$ for women to achieve equivalent breath alcohol concentrations (BrACs) across sex [60-62]. Ethanol beverages were served in a $10 \%$ solution by volume with the participants' preferred fruit juice flavor (cranberry or orange). The placebo beverage consisted of the fruit juice plus $3 \mathrm{ml}$ ethanol added as a taste mask. Mood, vital signs, and BrAC were recorded and measures of subjective response completed every $30 \mathrm{~min}$ postbeverage administration for $2.5 \mathrm{~h}$. Sessions ended at 8:00 pm, after confirmation that BrAC had fallen below $40 \mathrm{mg} / 100 \mathrm{ml}$ (as per NIAAA guidelines), and their blood pressure and heart rate had returned to baseline.

\section{Measures}

Subjective responses to alcohol were assessed using the Biphasic Alcohol Effects Scale (BAES; [63]) and Drug Effects Questionnaire (DEQ; [64]). BAES is a 14-item measure on which participants rate the degree to which they feel subjective stimulant (e.g., talkative, elated) and sedative (e.g., sedated, sluggish) responses to alcohol on 11-point Likert-type scales. Individual responses for the seven stimulation items and seven sedation items are summed to provide stimulation and sedation scale scores (score range $=$ $0-70$ ). DEQ consists of questions in a visual analog scale (score range $=0-100$ ) that measure the extent to which participants feel a drug effect, whether they like the drug effect, and if they would want to take more of the drug if given the choice. The participants' scores on the two alcohol- and placebo-sessions were averaged [59], and peak change scores were calculated (highest or lowest score after beverage administration minus prebeverage). The peak change difference scores for BAES 'Sedation' and 'Stimulation', and DEQ 'Feel', 'Like', and 'Want More' scales (average alcohol peak change score minus average placebo peak change score) were used in the subsequent analyses.

\section{fMRI reward paradigm}

To index neural reactivity to monetary rewards and losses, participants completed the reward-guessing game, the 'Doors' task [65]. Participants viewed a pair of doors on the screen and were told that behind one of the doors there was a monetary prize of $\$ 0.50(\uparrow)$ while behind the other door there was a loss of $\$ 0.25$ $(\downarrow)$. They were asked to choose one of the two doors using a button box to either win or lose money for each trial. They were told they had a chance of winning between $\$ 0$ and $\$ 15.00$ at the end of the task depending on their performance. Unbeknownst to participants, the task was rigged, such that behavior had no impact on actual outcomes and therefore was not analyzed or reported. The task consisted of 30 predetermined Wins and 30 Losses presented in pseudorandom order over two runs. The task lasted for $15 \mathrm{~min}$ and is based on a task used in previous studies [66-68].

\section{Acquisition and analysis of neuroimaging data}

Participants were scanned at the UIC Center for Magnetic Resonance Research using a $3.0 \mathrm{~T}$ GE scanner with an eight- channel phase-array radio frequency head coil. Functional images were acquired using gradient-echo echo-planar imaging (EPl; $2 \mathrm{~s}$ TR, $25 \mathrm{~ms} \mathrm{TE}, 82^{\circ}$ flip, $64 \times 64$ matrix, $200 \mathrm{~mm}$ FOV, $3 \mathrm{~mm}$ slice thickness, $0 \mathrm{~mm}$ gap, with 44 axial slices). Imaging data were inspected for high quality and scan stability; any individual with $>2$ $\mathrm{mm}$ displacement in any direction was excluded from the analyses. For excessive motion, fMRI time points that were severely affected by motion were removed using a 'scrubbing' approach in FSL (https://fsl.fmrib.ox.ac.uk/fsl/fslwiki/FSLMotionOutliers), with a root mean square signal change (DVARs) threshold of $0.5 \%$ and a frame-wise displacement threshold of $0.5 \mathrm{~mm}$ [69]. Preprocessing of fMRI data was carried out using Statistical Parametric Mapping software (SPM8, Wellcome Department of Imaging Neuroscience, London, UK). Images were spatially realigned to correct for head motion, slice-time corrected (44 slices, $\mathrm{TR}=2, \mathrm{TA}=2$, slice order: ascending interleaved, reference slice 21), warped to standardized Montreal Neurological Institute (MNI) space using the participants' mean functional image, resampled to $2 \mathrm{~mm}^{3}$ voxels, and smoothed with a $5 \mathrm{~mm}^{3}$ kernel to minimize noise and residual differences in gyral anatomy. The general linear model was applied to the time series, convolved with the canonical hemodynamic response function and with a $128 \mathrm{~s}$ high-pass filter. Condition effects were modeled with event-related regressors representing the occurrence of Win or Loss. Effects were estimated at each voxel, and for each subject. Individual contrast maps for Win > Loss were created for each person. Individual motion parameter files were included in the first level models as regressors-of-no-interest.

To test our hypotheses, contrast maps for Win > Loss were entered into second-level, one-sample $t$-test in SPM. To confirm that the task successfully activated reward-related regions during Win > Loss trials, we examined task activation across all subjects. As our hypotheses were specific to reward-related regions, neural activity from these models were considered significant if it exceeded cluster-based significance thresholding adjustment for multiple comparisons across a functional reward uniformity mask downloaded from the neurosynth toolbox (www.neurosynth.org; [70]) by searching the term 'reward' (Fig. S1). Based on simulations $(10,000$ iterations) performed using $3 \mathrm{dFWHMx}$ and $3 \mathrm{dCl}$ (ustSim with the autocorrelation function (updated and 'bug-free' on February 2019; [https://afni-nimh-nih-gov.proxy.cc.uic.edu/pub/ dist/doc/program_help/3dClustSim.html]; [71]), correction at $a<$ 0.05 is achieved with a voxel threshold of $p<0.001$ and a cluster size of at least 32 contiguous voxels (volume $=256 \mathrm{~mm}^{3}$ ). Next, to test the association between subjective response to drug and neural activity, we carried out bivariate Pearson correlations between BAES and DEQ scales [i.e., peak change difference scores] and extracted blood-oxygen-level-dependent (BOLD) parameter estimates (i.e., $\beta$-weights [arbitrary units]) from $10 \mathrm{~mm}$ (radius) spheres surrounding significant peak activations within neurosynth reward mask during Win > Loss. All tests were two-tailed and were deemed statistically significant at Bonferroni corrected $a<0.005$. Statistical analyses were performed and graphed using $\mathrm{R}$ software (Version 3.5.2; R Development Core Team, Vienna, 2004) and the following R-packages: sjPlot (Version 2.6.2; [72]) and ggplot2 (Version 3.1.0; [73]).

\section{RESULTS}

Task activation during reward

Reward receipt relative to loss (Win > Loss) significantly activated mesolimbic reward regions, including left and right ventral caudate and the NAcc, and left occipital gyrus (Fig. 1a, Table 2). The BOLD parameter estimates extracted from the left and right ventral caudate/NAcc clusters were highly correlated $(r=0.96, p<$ 0.001 ), and since it is unclear whether reward function is lateralized [74], we averaged these extracted estimates to create a single bilateral ventral caudate/NAcc (i.e., ventral striatum) value for each participant and used that in the subsequent analyses. 

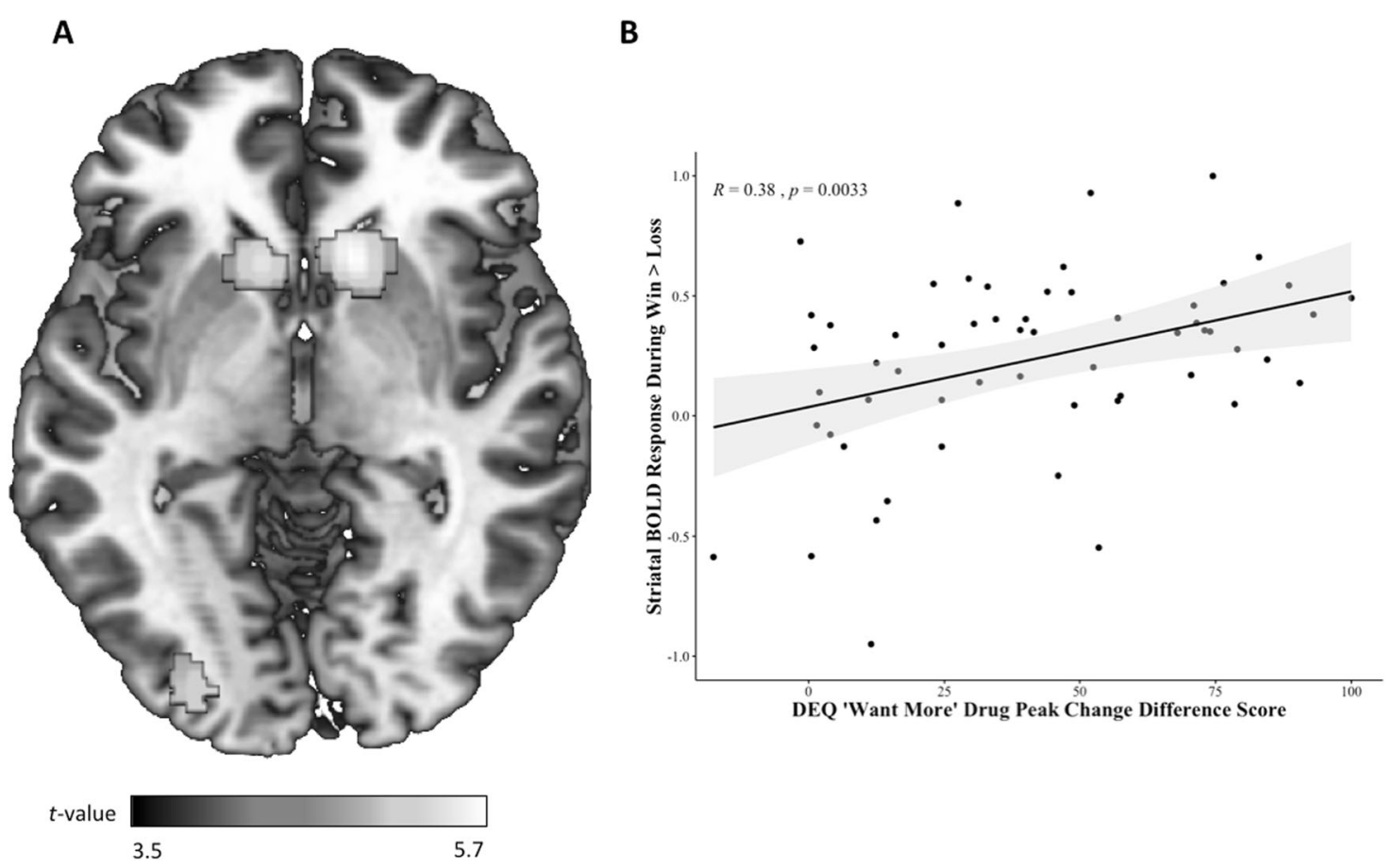

Fig. 1 Neural activation during reward task and its relation to subjective response to alcohol. a Task activation during Win relative to Loss trials within the neurosynth reward mask $(a<0.05$, corrected) across all participants. b Scatter plot depicting the significant correlation between DEQ 'Want More' drug peak change difference score and striatal BOLD response during Win relative to Loss trials across all participants. DEQ Drug Effects Questionnaire.

Table 2. Task activation during reward (Win $>$ Loss) within the neurosynth reward mask.

\begin{tabular}{|c|c|c|c|c|c|c|}
\hline Region & \multicolumn{3}{|c|}{ MNI coordinates } & Cluster (voxels) & Volume $\left(\mathrm{mm}^{3}\right)$ & $Z$ score \\
\hline Right ventral caudate/NAcc & 14 & 18 & -4 & 384 & 3072 & 5.67 \\
\hline Left ventral caudate/NAcc & -10 & 16 & -4 & 292 & 2336 & 5.42 \\
\hline Left occipital gyrus & -26 & -90 & -6 & 53 & 424 & 4.22 \\
\hline
\end{tabular}

Table 3. Differences in subjective responses to alcohol and placebo.

\begin{tabular}{lccc}
\hline & $\begin{array}{l}\text { Alcohol } \\
\text { mean (SD) }\end{array}$ & $\begin{array}{l}\text { Placebo mean } \\
(S D)\end{array}$ & Paired $t$-test \\
\hline $\begin{array}{l}\text { BAES } \\
\text { 'Stimulation' }\end{array}$ & $1.0(16.3)$ & $-7.5(9.9)$ & $t(57)=4.6, p<0.001$ \\
BAES 'Sedation' & $12.7(13.2)$ & $4.8(9.3)$ & $t(57)=4.1, p<0.001$ \\
DEQ 'Feel Drug' & $66.1(21.7)$ & $17.6(18.8)$ & $t(57)=17.5, p<0.001$ \\
DEQ 'Like Drug' & $75.1(15.8)$ & $27.4(24.0)$ & $t(57)=15.9, p<0.001$ \\
$\begin{array}{l}\text { DEQ 'Want } \\
\text { More Drug' }\end{array}$ & $68.5(25.5)$ & $27.5(26.1)$ & $t(57)=10.4, p<0.001$ \\
\hline
\end{tabular}

BAES Biphasic Alcohol Effects Scale, DEQ Drug Effects Questionnaire.

Subjective effects of alcohol

Alcohol significantly increased ratings of 'Stimulation' and 'Sedation' as well as 'Like', 'Feel', and 'Want More' ratings, relative to placebo (Table 3). Individuals differed in their responses to alcohol, with peak change difference scores (alcohol minus placebo) ranging from -23.5 to 42.0 for 'Stimulation', -32.0 to
47.0 for 'Sedation', 1.0 to 97.5 for 'Feel', 9.0 to 95.5 for 'Like', and -17.5 to 100.0 for 'Want More' scales.

Association between neural activation during reward and subjective response to alcohol: ROI analyses

BOLD parameter estimates extracted from the bilateral ventral caudate/NAcc during Win > Loss positively correlated with DEQ 'Like' and 'Want More' ratings, while left occipital gyrus negatively correlated with the DEQ 'Feel' ratings (i.e., peak change difference scores; Table 4). However, only the correlation between bilateral ventral caudate/NAcc and DEQ 'Want More' ratings passed Bonferroni correction $(p<0.005$; Fig. 1b). No area within the neurosynth reward mask during Win > Loss showed a significant correlation with the BAES 'Stimulation' and 'Sedation' ratings $\left(p_{\mathrm{s}}>0.51\right)$.

Exploratory analyses

To test the relative specificity of findings to DEQ 'Want More' ratings, we explored whether the association between DEQ 'Want More' ratings and peak BOLD signal response from the bilateral ventral caudate/NAcc remained significant when controlling for DEQ 'Like' ratings using partial correlation analysis. This correlation 
Table 4. Correlations between brain and subjective response measures.

\begin{tabular}{|c|c|c|c|c|c|c|c|}
\hline & 1. & 2. & 3. & 4. & 5. & 6. & 7. \\
\hline 2. BAES 'Sedation' PCDS & -0.16 & 1.0 & & & & & \\
\hline 4. DEQ 'Like Drug' PCDS & -0.05 & -0.09 & 0.26 & 1.0 & & & \\
\hline 5. DEQ 'Want More Drug' PCDS & -0.02 & 0.05 & 0.19 & $0.68^{* * *}$ & 1.0 & & \\
\hline
\end{tabular}

remained significant $(r=0.28, p=0.03)$, suggesting that the finding was specific to 'wanting' more alcohol (i.e., independent of the 'liking' of alcohol effects).

In addition, to better understand whether Win or Loss events contributed to the association between DEQ 'Want More' ratings and neural activation during Win $>$ Loss, we examined whether Win > Fixation and Loss > Fixation were separately related to DEQ 'Want More' ratings. For these exploratory analyses, individual DEQ 'Want More' scores were entered as a regressor of interest for the Win > Fixation and Loss > Fixation models. Neither model was significant indicating that the original findings were due to the relative difference between Wins vs. Losses.

Finally, to determine whether any of the participants' responses were related to prior drug use, we examined the relationships among alcohol-induced 'Want more' ratings, extracted BOLD parameter estimates of peak activation during Win $>$ Loss, and current and lifetime substance use measures. Neither alcoholinduced 'Want more' ratings nor BOLD peak activation during Win $>$ Loss were related to current (past month) alcohol, cigarette, caffeine, or marijuana use (Pearson correlations $p$ values $>0.005$ ).

\section{DISCUSSION}

The results of the present study show that individual differences in responses to drug and nondrug rewards are linked, such that greater striatal activation to nondrug (monetary) reward receipt in a drug-free state was associated with greater self-reported ratings of 'wanting more' alcohol after a single moderate dose of alcohol during a separate behavioral phase of the study. Specifically, the relative difference in the striatal activation between Win and Loss events, rather than during Win or Loss events separately, was significantly related to individual differences in the subjective response to alcohol. It has previously been reported that acute administration of a drug (or other rewarding stimulus) increases the desire for more ('priming' effects; [75-77]). Thus, the increase in ratings of 'wanting' more alcohol in the present study may reflect the induction of motivation for more of the drug, which appears to be a part of the rewarding effect of a drug. These findings provide the first direct evidence for a relationship between neural correlates of monetary reward receipt (when individuals are not acutely intoxicated) and sensitivity to the subjective rewarding effects of alcohol in humans.

The observed relationship is consistent with what is known about the neural basis of the 'incentive salience' theory of addiction. Proponents of this theory argue that motivational component of reward processing (i.e., wanting) is primarily mediated via increased dopamine neurotransmission within the mesolimbic reward circuitry, including the NAcc, caudate and other parts of the striatum [43, 78, 79]. Interestingly, our findings are consistent with the suggestion that the striatum in drug and nondrug reward processing appears to be related more to the saliency of the reward than to hedonic feelings (i.e., liking) associated with its consumption [80-84]. Specifically, we show that the association between ventral caudate/NAcc activation and reported motivation to consume more alcohol was independent of the reported liking of alcohol effects. Consistent with this, neuroimaging studies indicate that changes in striatal dopamine neurotransmission in humans are related more to subjective ratings of wanting drug and nondrug rewards, than to liking the drug [85-88]. Taken together, these studies suggest that dopamine signaling in the ventral striatum may guide addictive behaviors via increased attribution of incentive salience and motivation to acquire rewards, regardless of the pleasure experienced when the reward is ultimately consumed.

Of note, we did not observe a relationship between neural activation to monetary reward and alcohol-induced stimulation and sedation effects. One explanation may be that, given our a priori restriction of the analyses to the mesolimbic reward circuit, we did not select the right regions of interest to identify associations with self-reported ratings of stimulation and sedation. Alternatively, it is possible that the lack of findings may be due to the relatively small magnitude of alcohol-induced stimulation and sedation effects. This may in part be because of the experimental setup-the participants were alone in the testing rooms throughout the session with minimal social interaction. While a controlled environment is important for minimizing potentially confounding variables, it will be important for future studies to replicate present findings in more 'real-world' drinking settings where participants may experience greater stimulating and sedating effects of alcohol.

Previous substance use was not related to alcohol-induced motivation (i.e., wanting) to consume more alcohol or to peak neural activation during monetary reward receipt (Win $>$ Loss). This suggests that the observed relationship between alcohol and nondrug reward reflects stable, individual differences that are not the result of prior substance use. Therefore, a proportion of relatively light to moderate social drinkers in our sample may be at elevated risk for future onset of alcohol use problems, especially because we selected the age range to be before/during typical age of alcohol abuse expression. This relationship may be even stronger among individuals with alcohol use problems. Alternatively, some individuals in our sample may indeed be at risk for AUD, but were protected by other factors that preclude excessive alcohol use. Future longitudinal studies are needed to test these preliminary hypotheses.

The results of this study fit with the broader literature and contribute to a more complete understanding of addiction with important implications for prevention and treatment. Specifically, prior work has shown that individuals in the very early stages of substance addiction exhibit increased activation in the ventral 
striatum during monetary reward receipt compared with healthy controls [89]. Here, we show that a broad, reward-modality independent hypersensitive striatum may even predate the early stages of AUD, and constitute a risk factor for AUD. Consequently, we may be able to use neural activation to monetary reward to detect this broader trait early on, before alcohol use problems have developed. The use of monetary rewards in lieu of alcohol to indirectly measure brain reward function in the target reward regions that are typically affected by alcohol will also help avoid ethical issues of using alcohol as reinforcer in humans. This may also be particularly useful in adolescent populations, in whom it is not possible to study the behavioral and neural responses to acute alcohol intoxication.

The present findings should be interpreted in light of several limitations. First, we administered a single moderate dose of alcohol, and thus, it will be important for future studies to examine whether neural activation during monetary reward predicts the rewarding effects of alcohol in a dose-dependent manner. Second, our participants were healthy, moderate social drinkers who occasionally engaged in binge drinking, and it is therefore unclear whether the present findings would generalize to individuals with lighter or heavier drinking patterns. Third, we did not assess family history of alcohol and drug use, and thus could not account for its effect on the brain reward circuitry in our analyses. In addition, the relatively small sample size precluded our ability to explore potential moderators of interest, including sex and patterns of alcohol consumption, and perhaps detect significant effects. Future studies with larger samples will be needed to investigate these associations. There were also a couple of limitations related to the fMRI task. Although we attempted to examine the independent effects of Win and Loss events using Fixation, it is important to note that 'Fixation' is not a comparable control condition. In other words, the Doors task did not have a neutral condition in which participants neither gain nor lose money. Future studies should therefore consider incorporating a true control ('neutral') condition in order to determine the respective contributions of Win and Loss outcomes. The task also did not allow us to examine neural response during reward anticipation (i.e., outcome expectancy), making it difficult to compare our findings to other studies of risk. Finally, given the cross-sectional nature of our study, we did not collect measures of substance use over time. Future longitudinal studies are needed to determine whether acute alcohol responses or neural responses to reward predict development of problematic alcohol use.

In conclusion, our study provides the first direct evidence that measures of alcohol reward are related to neural indices of monetary reward. That is, individuals who report wanting more alcohol after a single moderate dose of alcohol also exhibited greater ventral striatal activation upon receipt of a monetary reward (without alcohol). These results provide evidence that individual differences in responses to drug and nondrug reward are linked, and together form a risk profile for AUD, particularly in young adults.

\section{FUNDING AND DISCLOSUR}

This publication was funded by the National Institute on Drug Abuse (NIDA; R01DA002812, Pls: HdW and KLP). SMG and JW were supported by the National Institute on Alcohol Abuse and Alcoholism (NIAAA; K23AA025111, PI: SMG; K01AA024519, PI: JW). Its contents are solely the responsibility of the authors and do not necessarily represent the official views of NIDA, NIAAA, or the National Institutes of Health. Authors declare no competing interests.

\section{AUTHOR CONTRIBUTIONS}

$\mathrm{HdW}$ and KLP were the principal investigators of the study. HdW, KLP, and SAL contributed to the conceptual design of the study, made important contributions to the editing of the paper and assisted in data analysis and interpretation. MR conducted the statistical analyses, interpreted the data, and wrote the initial draft of the paper. NAC, SMG, and JW assisted in paper preparation.

\section{ADDITIONAL INFORMATION}

Supplementary Information accompanies this paper at (https://doi.org/10.1038/ s41386-020-0728-6)

Publisher's note Springer Nature remains neutral with regard to jurisdictional claims in published maps and institutional affiliations.

\section{REFERENCES}

1. Room R, Babor T, Rehm J. Alcohol and public health. Lancet. 2005;365:519-30.

2. Dawson DA, Grant BF. The "Gray area" of consumption between moderate and risk drinking. J Stud Alcohol Drugs. 2011;72:453-8.

3. Sudhinaraset M, Wigglesworth $C$, Takeuchi DT. Social and cultural contexts of alcohol use: Influences in a social-ecological framework. Alcohol Res Curr Rev. 2016;38:35-45.

4. Morean ME, Corbin WR. Subjective response to alcohol: a critical review of the literature. Alcohol Clin Exp Res. 2010;34:385-95.

5. Ray LA, MacKillop J, Monti PM. Subjective responses to alcohol consumption as endophenotypes: advancing behavioral genetics in etiological and treatment models of alcoholism. Subst Use Misuse. 2010;45:1742-65.

6. King AC, McNamara PJ, Hasin DS, Cao D. Alcohol challenge responses predict future alcohol use disorder symptoms: a 6-year prospective study. Biol Psychiatry. 2014;75:798-806.

7. Schuckit MA. Low level of response to alcohol as a predictor of future alcoholism. Am J Psychiatry. 1994;151:184-9.

8. King AC, De Wit H, McNamara PJ, Cao D. Rewarding, stimulant, and sedative alcohol responses and relationship to future binge drinking. Arch Gen Psychiatry. 2011;68:389-99.

9. Koob GF, Volkow ND. Neurocircuitry of addiction. Neuropsychopharmacology. 2010;35:217-38.

10. Koob GF, Volkow ND. Neurobiology of addiction: a neurocircuitry analysis. Lancet Psychiatry. 2016;3:760-73.

11. Lüscher C, Malenka RC. Drug-evoked synaptic plasticity in addiction: from molecular changes to circuit remodeling. Neuron.2011;69:650-63.

12. Di Chiara G. Alcohol and dopamine. Alcohol Health Res World. 1997;21:108-14.

13. Clarke R, Adermark L. Dopaminergic regulation of striatal interneurons in reward and addiction: focus on alcohol. Neural Plast. 2015;2015:1-11.

14. Charlet $K$, Beck A, Heinz A. The dopamine system in mediating alcohol effects in humans. Curr Top Behav Neurosci. 2011;13:461-88.

15. Tupala E, Tiihonen J. Dopamine and alcoholism: neurobiological basis of ethanol abuse. Prog Neuro-Psychopharmacol Biol Psychiatry. 2004;28:1221-47.

16. Ramchandani VA, Umhau J, Pavon FJ, Ruiz-Velasco V, Margas W, Sun H, et al. A genetic determinant of the striatal dopamine response to alcohol in men. Mol Psychiatry. 2011;16:809-17.

17. Urban NBL, Kegeles LS, Slifstein M, Xu X, Martinez D, Sakr E, et al. Sex differences in striatal dopamine release in young adults after oral alcohol challenge: a positron emission tomography imaging study with [11C]raclopride. Biol Psychiatry. 2010;68:689-96.

18. Boileau I, Assaad JM, Pihl RO, Benkelfat C, Leyton M, Diksic M, et al. Alcohol promotes dopamine release in the human nucleus accumbens. Synapse. 2003:49:226-31.

19. Setiawan E, Pihl RO, Dagher A, Schlagintweit H, Casey KF, Benkelfat C, et al. Differential striatal dopamine responses following oral alcohol in individuals at varying risk for dependence. Alcohol Clin Exp Res. 2014;38:126-34.

20. Yoder KK, Albrecht DS, Dzemidzic M, Normandin MD, Federici LM, Graves T, et al. Differences in IV alcohol-induced dopamine release in the ventral striatum of social drinkers and nontreatment-seeking alcoholics. Drug Alcohol Depend. 2016;160:163-9.

21. Kegeles LS, Horga G, Ghazzaoui R, Rosengard R, Ojeil N, Xu X, et al. Enhanced striatal dopamine release to expectation of alcohol: a potential risk factor for alcohol use disorder. Biol Psychiatry Cognit Neurosci Neuroimaging. 2018;3:591-8.

22. Yoder KK, Constantinescu CC, Kareken DA, Normandin MD, Cheng T-E, O'Connor SJ, et al. Heterogeneous effects of alcohol on dopamine release in the striatum: a PET study. Alcohol Clin Exp Res. 2007;31:965-73.

23. Yoder KK, Kareken DA, Seyoum RA, O'Connor SJ, Wang C, Zheng Q-H, et al. Dopamine D2 receptor availability is associated with subjective responses to alcohol. Alcohol Clin Exp Res. 2005;29:965-70.

24. Gilman JM, Ramchandani VA, Davis MB, Bjork JM, Hommer DW. Why we like to drink: a functional magnetic resonance imaging study of the rewarding and anxiolytic effects of alcohol. J Neurosci. 2008;28:4583-91. 
25. Gilman JM, Ramchandani VA, Crouss T, Hommer DW. Subjective and neural responses to intravenous alcohol in young adults with light and heavy drinking patterns. Neuropsychopharmacology. 2012;37:467-77.

26. Weafer J, Ross TJ, O'Connor S, Stein EA, de Wit H, Childs E. Striatal activity correlates with stimulant-like effects of alcohol in healthy volunteers. Neuropsychopharmacology. 2018;43:2532-8.

27. Sescousse G, Caldú X, Segura B, Dreher JC. Processing of primary and secondary rewards: a quantitative meta-analysis and review of human functional neuroimaging studies. Neurosci Biobehav Rev. 2013;37:681-96.

28. Olsen CM. Natural rewards, neuroplasticity, and non-drug addictions. Neuropharmacology. 2011;61:1109-22.

29. McCabe C, Mishor Z, Cowen PJ, Harmer CJ. Diminished neural processing of aversive and rewarding stimuli during selective serotonin reuptake inhibitor treatment. Biol Psychiatry. 2010;67:439-45

30. Berns GS, McClure SM, Pagnoni G, Montague PR. Predictability modulates human brain response to reward. J Neurosci. 2001;21:2793-8.

31. McClure SM, Berns GS, Montague PR. Temporal prediction errors in a passive learning task activate human striatum. Neuron. 2003;38:339-46.

32. Pagnoni G, Zink CF, Montague PR, Berns GS. Activity in human ventral striatum locked to errors of reward prediction. Nat Neurosci. 2002;5:97-8.

33. Arnow BA, Desmond JE, Banner LL, Glover GH, Solomon A, Polan ML, et al. Brain activation and sexual arousal in healthy, heterosexual males. Brain. 2002;125:1014-23.

34. Breiter HC, Aharon I, Kahneman D, Dale A, Shizgal P. Functional imaging of neural responses to expectancy and experience of monetary gains and losses. Neuron. 2001;30:619-39.

35. Delgado MR, Stenger VA, Fiez JA. Motivation-dependent responses in the human caudate nucleus. Cereb Cortex. 2004;14:1022-30.

36. Delgado MR, Nystrom LE, Fissell C, Noll DC, Fiez JA. Tracking the hemodynamic responses to reward and punishment in the striatum. J Neurophysiol. 2000;84:3072-7.

37. Elliott R, Friston KJ, Dolan RJ. Dissociable neural responses in human reward systems. J Neurosci. 2000;20:6159-65.

38. Izuma K, Saito DN, Sadato N. Processing of social and monetary rewards in the human striatum. Neuron. 2008;58:284-94.

39. Knutson B, Westdorp A, Kaiser E, Hommer D. FMRI visualization of brain activity during a monetary incentive delay task. Neuroimage. 2000;12:20-7.

40. Nieuwenhuis S, Heslenfeld DJ, von Geusau NJA, Mars RB, Holroyd CB, Yeung N. Activity in human reward-sensitive brain areas is strongly context dependent. Neuroimage. 2005;25:1302-9.

41. Thut G, Schultz W, Roelcke U, Nienhusmeier M, Missimer J, Maguire RP, et al. Activation of the human brain by monetary reward. Neuroreport. 1997;8:1225-8.

42. Zald DH, Boileau I, El-Dearedy W, Gunn R, McGlone F, Dichter GS, et al. Dopamine transmission in the human striatum during monetary reward tasks. J Neurosci. 2004;24:4105-12

43. Berridge $\mathrm{KC}$, Robinson TE. Liking, wanting, and the incentive-sensitization theory of addiction. Am Psychol. 2016;71:670-9.

44. Kelley $A E$, Berridge $K C$. The neuroscience of natural rewards: relevance to addictive drugs. J Neurosci. 2002;22:3306-11.

45. Goldstein RZ, Woicik PA, Moeller SJ, Telang F, Jayne M, Wong C, et al. Liking and wanting of drug and non-drug rewards in active cocaine users: the STRAP-R questionnaire. J Psychopharmacol. 2010;24:257-66.

46. Bustamante J-C, Barrós-Loscertales A, Costumero V, Fuentes-Claramonte P, RosellNegre $\mathrm{P}$, Ventura-Campos $\mathrm{N}$, et al. Abstinence duration modulates striatal functioning during monetary reward processing in cocaine patients. Addict Biol. 2014;19:885-94

47. Jia Z, Worhunsky PD, Carroll KM, Rounsaville BJ, Stevens MC, Pearlson GD, et al. An initial study of neural responses to monetary incentives as related to treatment outcome in cocaine dependence. Biol Psychiatry. 2011;70:553-60.

48. Bühler M, Vollstädt-Klein S, Kobiella A, Budde H, Reed $\sqcup$, Braus DF, et al. Nicotine dependence is characterized by disordered reward processing in a network driving motivation. Biol Psychiatry. 2010;67:745-52.

49. Wrase J, Schlagenhauf $F$, Kienast $T$, Wüstenberg $T$, Bermpohl F, Kahnt $T$, et al. Dysfunction of reward processing correlates with alcohol craving in detoxified alcoholics. Neuroimage.2007;35:787-94.

50. Heather Nick, Segal G. Addiction \& choice: rethinking the relationship. 1st ed. New York, NY: Oxford University Press; 2016

51. Crane NA, Gorka SM, Weafer J, Langenecker SA, De Wit H, Phan KL. Neural activation to monetary reward is associated with amphetamine reward sensitivity. Neuropsychopharmacology. 2018;43:1738-44.

52. Langenecker SA, Kling LR, Crane NA, Gorka SM, Nusslock R, Damme KSF, et al. Anticipation of monetary reward in amygdala, insula, caudate are predictors of pleasure sensitivity to d-Amphetamine administration. Drug Alcohol Depend. 2020;206:107725
53. Liu X, Hairston J, Schrier M, Fan J. Common and distinct networks underlying reward valence and processing stages: a meta-analysis of functional neuroimaging studies. Neurosci Biobehav Rev. 2011;35:1219-36.

54. Kirkpatrick MG, De Wit H. In the company of others: social factors alter acute alcohol effects. Psychopharmacol. 2013;230:215-26.

55. Weafer J, Gallo DA, de Wit H. Effect of alcohol on encoding and consolidation of memory for alcohol-related images. Alcohol Clin Exp Res. 2016;40:1540-7.

56. First MB, Williams JBW, Karg RSSR. Structured clinical interview for DSM-5research version (SCID-5 for DSM-5, Research Version; SCID-5-RV). Arlington, VA: American Psychiatric Association; 2015.

57. Weafer J, de Wit H, Weafer J, Phan KL. Poor inhibitory control is associated with greater stimulation and less sedation following alcohol. Psychopharmacology. 2019:1-8.

58. Rhodes JD, Hawk LW. Smoke and mirrors: the overnight abstinence paradigm as an index of disrupted cognitive function. Psychopharmacol. 2016;233:1395-404.

59. Murray $\mathrm{CH}$, Weafer J, de Wit $\mathrm{H}$. Stability of acute responses to drugs in humans across repeated testing: findings with alcohol and amphetamine. Drug Alcohol Depend. 2020:107989. (in press)

60. Sutker PB, Tabakoff B, Goist KC, Randall CL. Acute alcohol intoxication, mood states and alcohol metabolism in women and men. Pharmacol Biochem Behav. 1983;18:349-54.

61. Fillmore MT. Cognitive preoccupation with alcohol and binge drinking in college students: alcohol-induced priming of the motivation to drink. Psychol Addict Behav. 2001;15:325-32.

62. Doty $\mathrm{P}$, de Wit $\mathrm{H}$. Effect of setting on the reinforcing and subjective effects of ethanol in social drinkers. Psychopharmacol. 1995;118:19-27.

63. Martin CS, Earleywine M, Musty RE, Perrine MW, Swift RM. Development and validation of the biphasic alcohol effects scale. Alcohol Clin Exp Res. 1993;17:140-6.

64. Morean ME, de Wit H, King AC, Sofuoglu M, Rueger SY, O'Malley SS. The drug effects questionnaire: psychometric support across three drug types. Psychopharmacol. 2013;227:177-92.

65. Crane NA, Gorka SM, Weafer J, Langenecker SA, de Wit H, Phan KL. Preliminary evidence for disrupted nucleus accumbens reactivity and connectivity to reward in binge drinkers. Alcohol Alcohol. 2017;52:647-54.

66. Hajcak G, Moser JS, Holroyd CB, Simons RF. The feedback-related negativity reflects the binary evaluation of good versus bad outcomes. Biol Psychol. 2006;71:148-54.

67. Foti D, Hajcak G. Depression and reduced sensitivity to non-rewards versus rewards: evidence from event-related potentials. Biol Psychol. 2009;81:1-8.

68. Carlson JM, Foti D, Mujica-Parodi LR, Harmon-Jones E, Hajcak G. Ventral striatal and medial prefrontal BOLD activation is correlated with reward-related electrocortical activity: a combined ERP and fMRI study. Neuroimage. 2011;57:1608-16.

69. Power JD, Barnes KA, Snyder AZ, Schlaggar BL, Petersen SE. Spurious but systematic correlations in functional connectivity MRI networks arise from subject motion. Neuroimage. 2012;59:2142-54.

70. Yarkoni T, Poldrack RA, Nichols TE, Van Essen DC, Wager TD. Large-scale automated synthesis of human functional neuroimaging data. Nat Methods. 2011;8:665-70.

71. Cox RW. AFNI: software for analysis and visualization of functional magnetic resonance neuroimages. Comput Biomed Res. 1996;29:162-73.

72. Lüdecke D. sjplot: Data visualization for statistics in social science. 2018.

73. Wickham H. Ggplot2: elegrant graphics for data analysis. 2016.

74. Molochnikov I, Cohen D. Hemispheric differences in the mesostriatal dopaminergic system. Front Syst Neurosci. 2014;8:1-14.

75. De Wit H. Priming effects with drugs and other reinforcers. Exp Clin Psychopharmacol. 1996;4:5-10.

76. de Wit $\mathrm{H}$, Chutuape MA. Increased ethanol choice in social drinkers following ethanol preload. Behav Pharmacol. 1993;4:29-36.

77. Kirk JM, De Wit H. Individual differences in the priming effect of ethanol in social drinkers. J Stud Alcohol. 2000;61:64-71.

78. Pool E, Sennwald V, Delplanque S, Brosch T, Sander D. Measuring wanting and liking from animals to humans: a systematic review. Neurosci Biobehav Rev. 2016;63:124-42.

79. Robinson MJF, Fischer AM, Ahuja A, Lesser EN, Maniates H. Roles of "wanting" and "liking" in motivating behavior: gambling, food, and drug addictions. Curr Top Behav Neurosci. 2016;27:105-36.

80. Zink CF, Pagnoni G, Martin-Skurski ME, Chappelow JC, Berns GS. Human striatal responses to monetary reward depend on saliency. Neuron.2004;42:509-17.

81. Berridge $\mathrm{KC}$, Robinson TE, Aldridge JW. Dissecting components of reward: 'liking', 'wanting', and learning. Curr Opin Pharmacol. 2009;9:65-73.

82. Zink CF, Pagnoni G, Martin ME, Dhamala M, Berns GS. Human striatal response to salient nonrewarding stimuli. J Neurosci. 2003;23:8092-7. 
Striatal activation to monetary reward is associated with alcohol reward... M Radoman et al.

83. Leyton M, Casey KF, Delaney JS, Kolivakis T, Benkelfat C. Cocaine craving, euphoria, and self-administration: a preliminary study of the effect of catecholamine precursor depletion. Behav Neurosci. 2005;119:1619-27.

84. Hardman CA, Herbert VMB, Brunstrom JM, Munafò MR, Rogers PJ. Dopamine and food reward: effects of acute tyrosine/phenylalanine depletion on appetite. Physiol Behav. 2012;105:1202-7.

85. Evans AH, Pavese N, Lawrence AD, Tai YF, Appel S, Doder M, et al. Compulsive drug use linked to sensitized ventral striatal dopamine transmission. Ann Neurol. 2006;59:852-8.

86. Smith $\mathrm{CT}$, Dang LC, Cowan RL, Kessler RM, Zald DH. Variability in paralimbic dopamine signaling correlates with subjective responses to d-amphetamine. Neuropharmacology. 2016;108:394-402.
87. Volkow ND, Wang GJ, Fowler JS, Logan J, Jayne M, Franceschi D, et al. 'Nonhedonic' food motivation in humans involves dopamine in the dorsal striatum and methylphenidate amplifies this effect. Synapse. 2002;44: 175-80.

88. Leyton M, Boileau I, Benkelfat C, Diksic M, Baker G, Dagher A. Amphetamineinduced increases in extracellular dopamine, drug wanting, and novelty seeking: a PET/[11C]raclopride study in healthy men. Neuropsychopharmacology. 2002;27:1027-35.

89. Luijten M, Schellekens AF, Kühn S, MacHielse MWJ, Sescousse G. Disruption of reward processing in addiction: an image-based meta-analysis of functional magnetic resonance imaging studies. JAMA Psychiatry. 2017;74:387-98. 